\title{
SiDaCoS: Product and Process Models on Construction Sites
}

\author{
Jan Reinhardt ${ }^{1}$, James H. Garrett, Jr. $^{2}$, and Burcu Akinci ${ }^{3}$ \\ Carnegie Mellon University, Dept. of Civil and Environmental Engineering
}

\section{Summary}

Site superintendents performing project management tasks on construction sites need to access project documents and need to collect information that they observe while inspecting the site. Often, information that is observed on a construction site needs to be integrated into electronic documents or project control systems. In the future, we expect integrated product and process models to be the medium for storing and handling construction project management information. Even though mobile computing devices today are already capable of storing and handling such integrated product and process data models, the user interaction with such large and complex models is difficult and not adequately addressed in the existing research. In this paper, we introduce a system that supports project management tasks on construction sites effectively and efficiently by making integrated product and process models accessible. In order to effectively and efficiently enter or access information, site superintendents need visual representations of the project data that are flexible with respect to the level of detail, the decomposition structure, and the type of visual representation. Based on this understanding of the information and data collection needs, we developed the navigational model framework and the application Site Data Collection System (SiDaCoS), which implements that framework. The navigational model framework allows site superintendents to create customized representations of information contained in a product and process model that correspond to their data access and data collection needs on site.

Key words: data collection, construction management, product and process models, system architecture, mobile computing.

\section{Introduction}

In the last decade researchers proposed integrated product and process models for representing construction information during the design phase, and also during the construction phase of a building. Such models integrate the information that different participants create, share and use in the design phase and in the construction phase of a project (Froese et al. 1999; Staub-French and Fischer 2000). This integration of product and process information supports consistency checks of the model, creation of cross references between different types of design and process information, and rich graphical representations that support communication and decision making.

During the construction phase of a project, a large share of the information that needs to be established or exchanged, is observed or needed on the construction site. Site superintendents have to collect progress and quality related information and need product information and specifications while on a construction site. Mobile computers can make information contained in product and process models available right at the construction site and allow site superintendents

\footnotetext{
1 *Research Assistant,janr@andrew.cmu.edu,corresponding author,

${ }^{2}$ Professor of Civil Engineering, and

${ }^{3}$ Assistant Professor of Civil Engineering
} 
to enter observed conditions right on site without using paper based solutions in order to transfer information between the site and the site office.

To date, different systems make construction project management informatio $\mathrm{n}$ accessible on construction sites. Liu et al. (2002) propose a system that makes product and process information stored on a data server accessible on a mobile computing device that can be used on construction sites. Depending on the type of the mobile computing device, the system generates representations of the data contained in the product and process model. Similarly, Pena Mora and Dwivedi (2002) propose a server based collaboration system that allows project participants to collaborate by using mobile computers for exchanging product and process information.

The challenge in using integrated product and process models on mobile computers is the complexity of these models and the limited means of interaction of mobile computing devices. Product and process models can have tens of thousands of entities that site superintendents may have to access in order to enter or retrieve information. In our research we focused on progress monitoring and the creation and administration of punch lists. Both tasks are important project management tasks that involve data access and data collection on site (Boyle 1993, Saram and Ahmed 2001).

This paper gives a high level overview of the Site Data Collection System (SiDaCoS), a mobile computing system that supports efficient interaction with integrated product and process models on a mobile computer and elaborates on the user interaction with SiDaCoS. More detailed descriptions of the different aspects of our research are documented in Reinhardt 2003; Reinhardt, Akinci, and Garrett 2002; and Reinhardt, Akinci, and Garrett 2004.

\section{Interaction needs}

In order to understand the information and data collection needs of site superintendents on construction sites, we conducted case studies on three construction sites that varied in several dimensions. These case studies can be seen as a contextual inquiry, a technique in human computer interaction research for developing an understanding of the task and establishing system requirements (Beyer and Holtzblatt 1997). We identified that site superintendents need task specific information at different levels of detail and in different forms of representations (Reinhardt 2004). Figure 1 shows a part of a construction schedule and the corresponding section of an office building. In the case studies we identified that activities of a construction schedule typically pertain to sections of a building. However, in many cases site superintendents need to collect progress information at the building element level, room level, and at the project level. The case studies revealed that site superintendents need information representations at different levels of detail, and different inspection situations on construction sites require different visual representations. A site superintendent may identify a certain wing or a certain floor in a tree representation of a construction project decomposition, but he or she may require a $2 \mathrm{D}$ or 3D representation of the project in order to address individual building elements. Finally, we identified that different inspection situations on a construction site require different decompositions of the project. A site superintendent inspecting windows from inside the building may use a floor-wise decomposition of the windows of the project, whereas a façade-wise decomposition of the windows of the project may be required for a an inspection from outside a building. 
Providing a system with representations of information that are flexible with respect to level of detail, decomposition and type of representations, we hope to make interaction with mobile computing applications on construction sites effective and more efficient compared to existing systems.

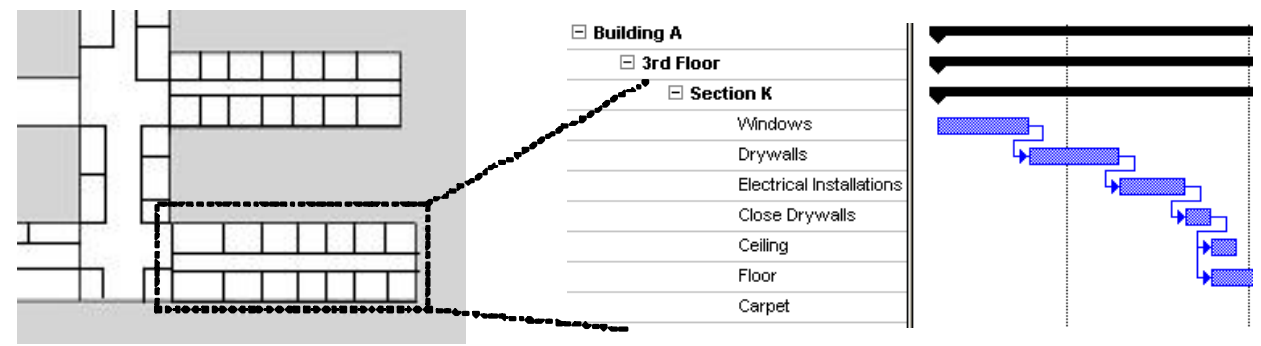

Figure 1. Typical decomposition of a construction schedule by floor and section of the building.

\section{SiDaCoS and the navigational model framework}

With the Site Data Collection System ( $\mathrm{SiDaCoS})$, we created a system that allows effective and efficient interaction with product and process models on construction sites. The design of $\mathrm{SiDaCoS}$ was driven by the information and data collection needs identified in the case studies. The core component of SiDaCoS is the navigational model framework. This framework is an extensible software component that supports the customization of representations of information contained in a product and process model. The navigational model framework consists of navigational models, ElementAmbassadors, and an implementation of the ReteAlgorithm (Forgy 1982).

Navigational models are customizable constructs that facilitate interaction with information contained in a product and process model at different levels of detail, by using different decomposition structures, and by presenting information in different types of representations. Navigational models group information contained in a product and process model into labeled sets that correspond to the tasks a site superintendent may wish to perform during an inspection of a construction site. These sets are organized in a tree structure. By addressing nodes of that tree structure, site superintendents can efficiently address the low level entities of information of a product and process model. Considering the tree structure representation of a navigational model depicted in Figure 2, users can very efficiently select all windows of the building by addressing the root node of the tree structure, yet they can also address individual windows at lower levels of detail. Since navigational models organize information contained in a product and process model they can be created and abandoned without changing the data contained in the entities of the product and process model. Different navigational models can organize the information contained in product and process models under specific aspects, such as specific tasks, or specific phases of the construction project (Reinhardt, Akinci, and Garrett 2002). Moreover, these entities can still be presented in their original representations, e.g. building elements as $3 \mathrm{D}$ representations and activities as bars in a construction schedule. 


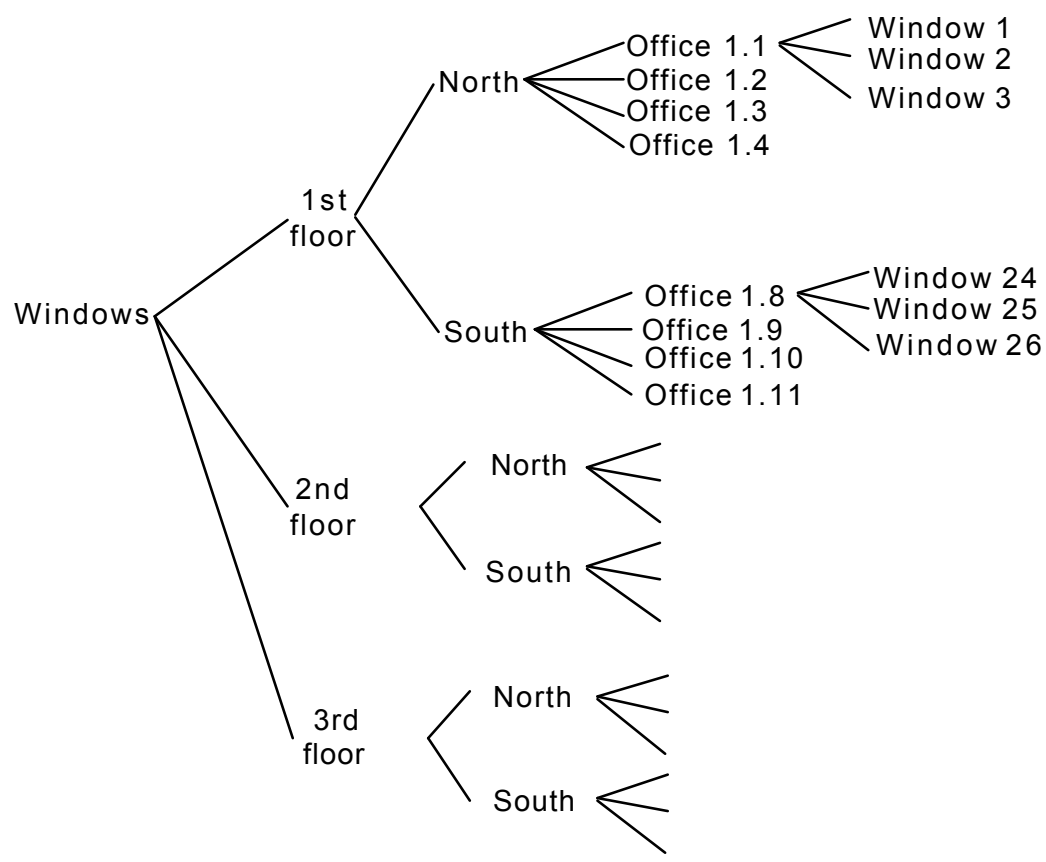

Figure 2. Tree representation of a navigational model

In some cases, the granularity of the information contained in the product and process model may not be sufficient for effective and efficient interaction. Wall elements, for example, may be modeled in a product and process model as one entity. However, if site superintendents inspect a building room by room, they may wish to address the single faces of a wall rather than the entire wall element. For this reason the navigational model framework provides ElementAmbassadors. ElementAmbassadors are disaggregatable constructs that act as proxies for entities of a product and process model. A second function of ElementAmbassadors is the propagation of state information between different representations of information contained in a product and process model. This synchronization is essential for efficient user interaction (Baldonado, Woodruff and Kuchinski 2000).

The third component of the navigational model framework, an implementation of the ReteAlgorithm (Forgy 1982), facilitates the efficient population of navigational models with element ambassadors. Based on features of entities of the product and process model or ElementAmbassadors, this mechanism performs matching and mapping operations between ElementAmbassadors and nodes of the navigational model. An in-depth description of this component is available in (Reinhardt 2003).

Figure 3 shows the relationship between navigational models, ElementAmbassadors and the product and process model. Each entity of the integrated product and process model is associated with one ElementAmbassador. In Figure 3, the first ElementAmbassador is disaggregated into two more detailed ElementAmbassadors in order to provide conceptual representations at the needed level of detail. The figure also shows how two navigational models organize the set of ElementAmbassadors, which represent the information contained in the product and process model. The first navigational model (1) constitutes a structure that better supports the inspection of a building from outside, whereas the second navigational model(2) better supports data access and data collection from inside a building. 


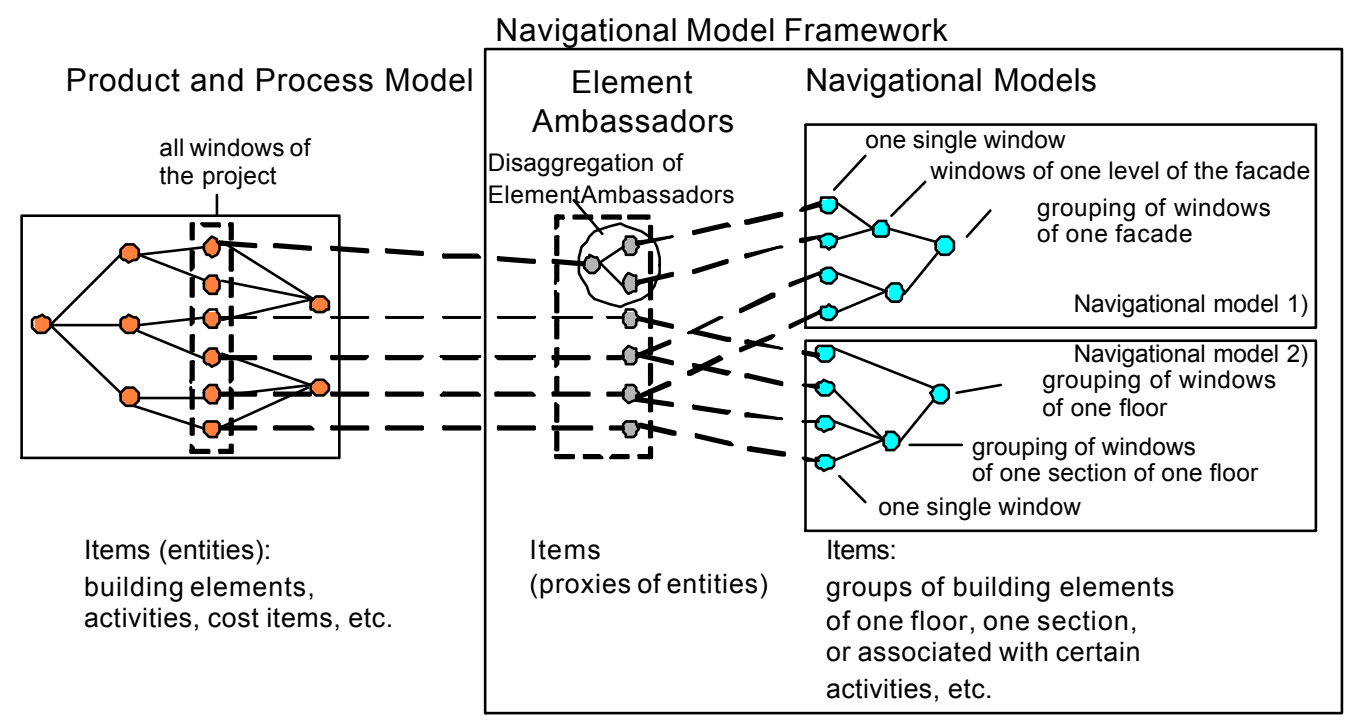

Figure 3. Relationship between entities of a product and process model, ElementAmbassadors and navigational models.

\section{User interface and interaction style}

The user interface of $\mathrm{SiDaCoS}$ has three main views. The first view, depicted in Figure 4a, shows a tree representation of a building project on the left hand side and a list of activities on the right hand side. This view supports progress monitoring, as the user can select building elements and activities, and can assign the selected elements and activities a construction progress by making the appropriate selection in the "Progress monitoring" menu. The second view, which supports the creation and administration of punch lists, is similar to the progress monitoring view. The left hand side shows a hierarchical decomposition of the project and the right hand side contains a table of punch list items. After the selection of the building element(s) to which a punch list item pertains, the user can open a dialog to describe the punch list item by selecting the appropriate menu item in the menu bar. The tree structures on the left hand side of these two views represent the navigational models that have been chosen for a particular data access and data collection task. Figure $4 \mathrm{~b}$ shows a $2 \mathrm{D}$ view of the building project. A site superintendent can use this type of representation in order to make selections of building elements that may be more difficult to make in a tree structure representation of the building.

Figure $4 \mathrm{c}$ shows the controls of $\mathrm{SiDaCoS}$ for navigation and selection, which allow quick switching between different types of representation, different levels of detail, and different decomposition structures. In order to switch between the three different views, and hence different types of representations of $\mathrm{SiDaCoS}$, the site superintendent can use the view switching tabs below the menu bar. By changing the specified level of detail in the level of detail control, site superintendents can control the granularity of elements that are picked if they make selection operations in the various views. The navigational model selection control supports the selection of an appropriate decomposition structure (see Figure $4 \mathrm{c}$ and $4 \mathrm{~d}$ ).

By using the selection mode control, site superintendents can choose the appropriate selection mode for selection operations of nodes of navigational models and individual ElementAmbassadors. The available selection modes are the following: (1) single selection mode, (2) multiple selection mode, (3) single navigational model selection mode (we elaborate 
on the navigational model selection node in the following paragraphs); and (4) multiple navigational model selection mode. Depending on the chosen selection mode, elements are selected or deselected if the user hits an element (building element, activity or punch list item). The single selection mode and the single navigational model selection mode correspond to the "Replace" selection operation of Wills' (1996) taxonomy of selection operations. If new selections are made, the elements that were selected before will be deselected. The multiple selection mode and the multiple navigational model selection mode correspond to the "Add" operation of Wills' (1996) taxonomy of selection operations.

The navigational model selection mode requires the user to define the level of detail on which he or she wants to interact with the data model. This level of detail is a numerical value that corresponds to the depth in the tree representation of a chosen (active) navigational model (see level of detail control in Figure 4c). If the user selects an element in any representation or view all sibling elements that the selected element has in the chosen navigational model at the specified level of detail are selected and represented as being selected in all representations. The navigational model selection mode therefore supports interaction at different levels of detail with representations other than tree representations. Figure 5 illustrates selection operations on different levels of detail that are performed in a 2D/3D representation of building elements by using the navigational model selection mode.

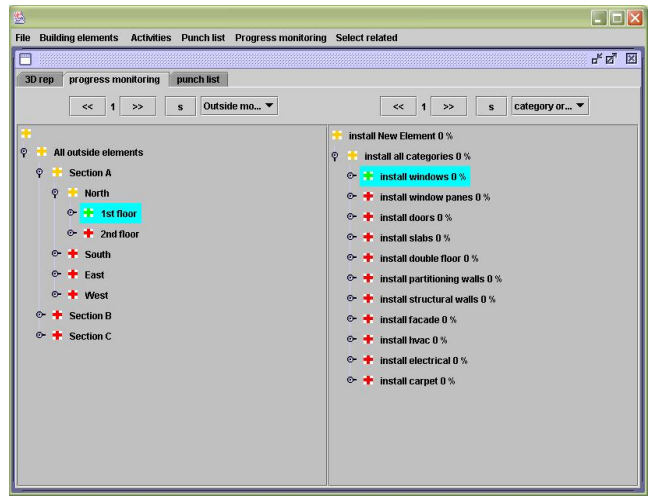

A) Tree representation of navigational models

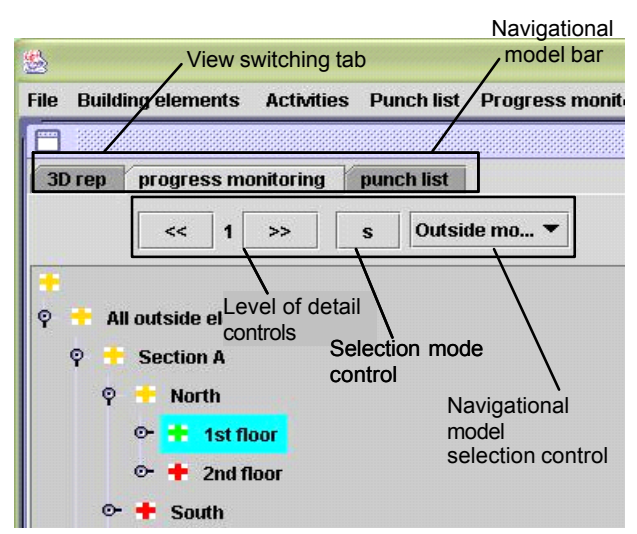

C) Controls for navigation and selection operations

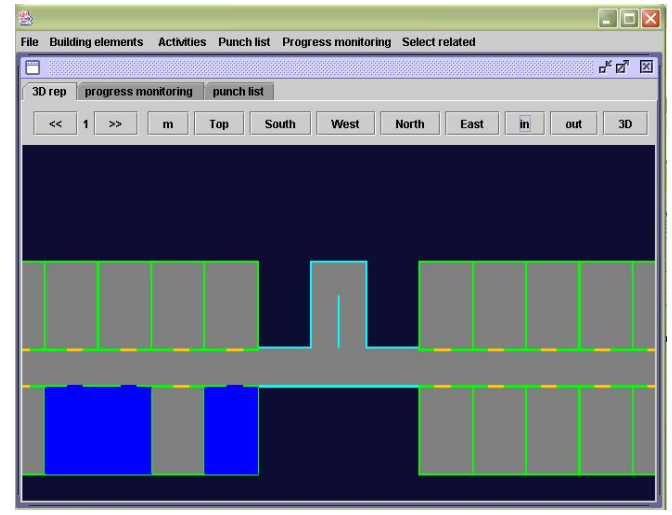

B) 2D representation of product model data

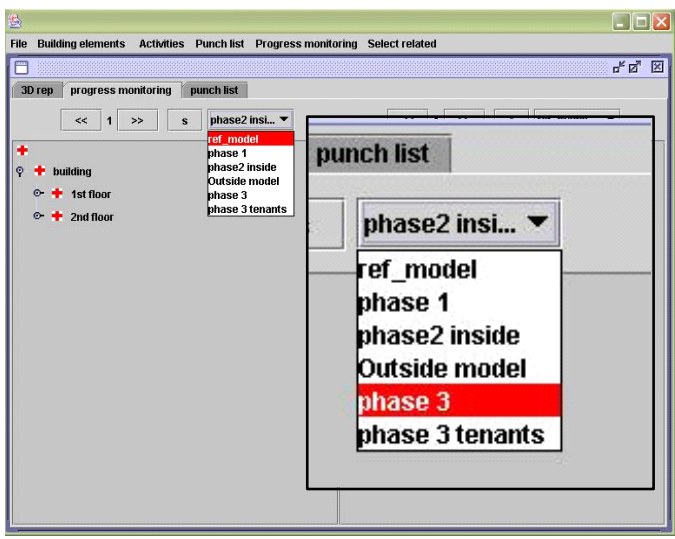

D) Selection of available navigational models

Figure 4. The User Interface of $\mathrm{SiDaCoS}$ 


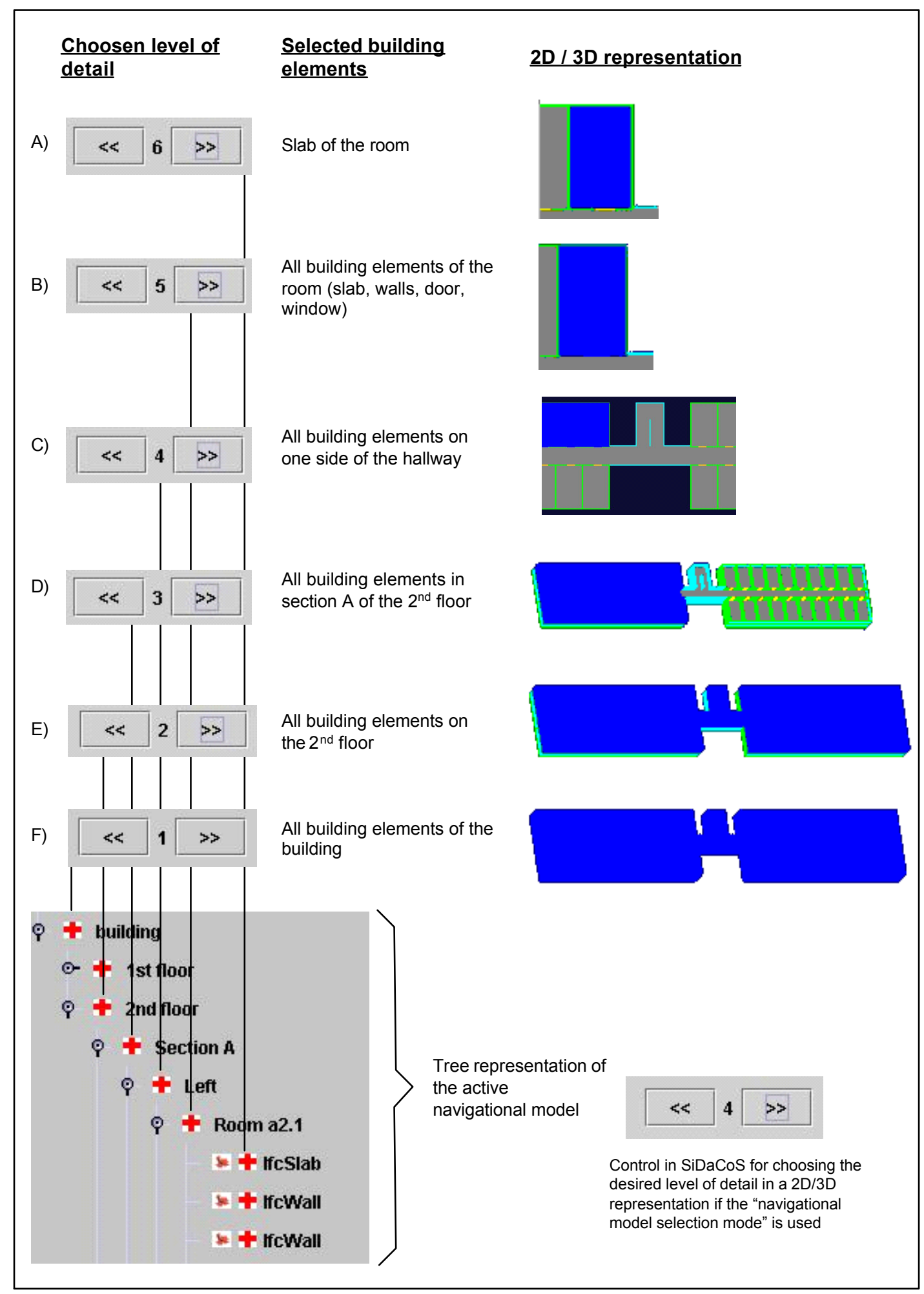

Figure 5. Illustration of the navigational model selection mode

\section{Usability and performance}

We validated $\mathrm{SiDaCoS}$ for the two project management tasks of progress monitoring and the creation and administration of punch lists. As a mobile computing platform, we used a tablet PC 
with a touch screen and a stylus. The validation of the software had to cover the usability of the user interface, the effectiveness and efficiency of the interaction process, the performance of the application, as well as an evaluation of the time needed for customizing the system for specific projects.

In order to validate the usability of the system, and the effectiveness and efficiency of the interaction, we performed user experiments and a Keystroke Level Model (KLM) analysis. Based on situations that were observed in case studies on construction sites, we defined a set of inspection situations. We asked test subjects to perform data access and data collection tasks with $\mathrm{SiDaCoS}$ and an existing system on a tablet PC for progress monitoring tasks. Moreover, we conducted a Keystroke Level Model analysis for more progress monitoring and punch list creation and administration tasks, and compared $\mathrm{SiDaCoS}$ with existing systems. Figure 6 shows the results of the KLM analysis, which correspond very well with the results of the user experiments (Reinhardt 2003). These results indicate that users can perform progress monitoring tasks (situations 1.1-2.4) more efficiently with $\mathrm{SiDaCoS}$ than with the existing system ICMMS (Reinhardt 2000). For tasks related to the creation and administration of punch lists (situations 3.1-3.5) we observed an efficiency that is comparable to the one of the existing systems PocketCad PunchList (ArcSecond Inc.). However, SiDaCoS could support data access and data collection for both project management tasks in all situations, whereas the alternative systems could not provide the needed support in all cases. Depending on the different systems that were tested, effective and efficient data access and data entry was only possible in $0 \%$ of the situations for the least effective system and in $87 \%$ of the considered situations for the most effective system (Reinhardt 2003). However, the efficiency of the interaction with the most effective alternative system was very low.

Performance times for the inspection situations using different system scenarios

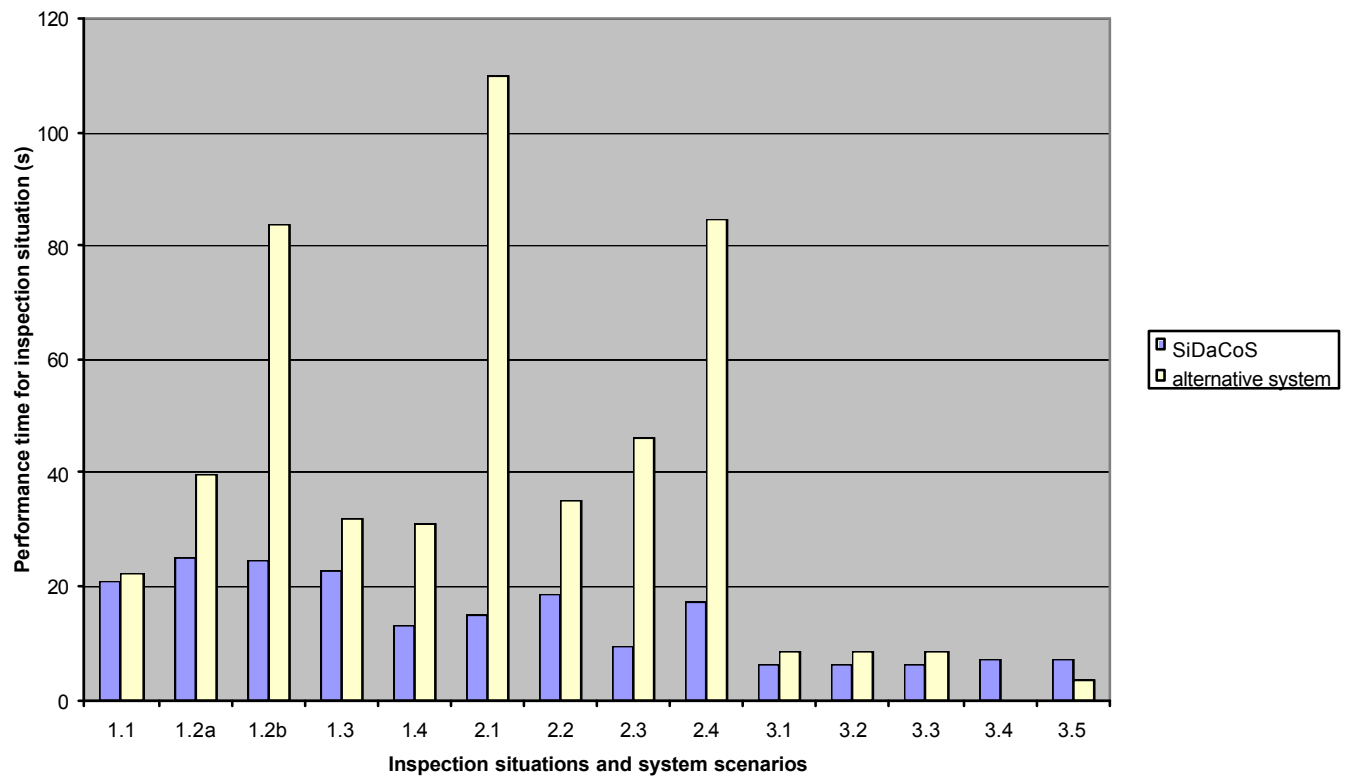

Figure 6. Performance time for performing interaction tasks with $\mathrm{SiDaCoS}$ and an alternative application (ICMMS). 
Furthermore, we demonstrated that for typical construction projects, the needed navigational models can be easily created and customized. The validation also showed that the creation of navigational models of complex and irregular projects can be very time consuming. However, due to the fact that navigational models are user-defined constructs, the user can always trade off the effort for creating specialized navigational models with the benefit of using these models.

\section{Summary and conclusions}

In this paper we have given a high level overview of $\mathrm{SiDaCoS}$, a system that makes integrated product and process models accessible on construction sites. The design of this system is based information and data collection needs of site superintendents on construction sites. We could validate that our design allows effective and efficient interaction with complex project management information on a mo bile computer.

$\mathrm{SiDaCoS}$ supports an interaction style with project management information that is similar to the interaction with paper based solutions in the field. The system provides site superintendents with flexibility with respect to the level of detail and the type of representation that is used in order to capture or access information. Due to the fact that $\mathrm{SiDaCoS}$, contrary to alternative applications, was effective in all considered test applications, we consider the system to be a true alternative to paper-based solutions. We see the elimination of paper-based data access and data collection solutions on construction sites and the ability to interact with product and process models on a construction sites as important steps toward the use of truly integrated product and process models in the construction process.

We see the concepts of navigational models that have been implemented in the navigational model framework as a powerful approach for interacting with product and process models. The fact that the navigational model framework supports both project management tasks, progress monitoring and the creation and administration of punch lists, suggests some generality of the approach. In the future, we would like to explore the applicability of the navigational model framework to other project management tasks on construction sites, and also in office settings. Moreover, we see research opportunities in the automated creation of navigational models.

\section{Acknowledgements}

We thank Hochtief AG, Essen, Germany, for providing funding and support for the research described in this paper.

\section{References}

Baldonado, M., Woodruff, A., and Kuchinsky, A. (2000). Guidelines for Using Multiple Views in Information Visualization, Proc. Advanced Vis. Interfaces 2000, Palermo, Italy, May 2000, pp. 110-119.

Beyer H. and Holtzblatt, K. (1998). Contextual Design: Defining Customer-Centered Systems. Morgan Kaufmann Publishers, Inc., San Francisco, CA.

Boyle, M.A., (1993). Taking the pain out of the punch list, Civil Engineering August 1993. 
Darshi de Saram, D. and Ahmed, S.M. (2001). Construction Coordination Activities: What is important and what consumes time, Journal of Management in Engineering Oct. 2001, 17 (4), pp. 185-238.

Forgy, C.L. (1982). Rete: A Fast Algorithm for the Many Pattern/ Many Object Pattern Match Problem. Artificial Intelligence 19, pp.17-37.

Froese, T., Fischer, M., Grobler, F., Ritzenthaler, Yu, K., Sutherland, S., Staub, S., Akinci, B., Akbas, R., Koo, B., Barron, A., and Kunz, J. (1999). Industry Foundation Classes for Project Management - A Trial Implementation, ITCON electronic journal, at http://www.itcon.org.

Liu, D., Cheng, J., Law, K.H., and Wiederhold, G. (2002). Ubiquitous Computing Environment for Project Management Services, Proceedings of the International Workshop on Information Technology in Civil Engineering, Washington, DC, pp. 273-285, 2002.

Peña-Mora, F. and Dwivedi, G. (2002). A Multiple Device Collaborative and Real Time Analysis System for Project Management in Civil Engineering, to ASCE Journal of Computing in Civil Engineering, 2002.

Reinhardt, J., James H. Garrett Jr., and Raimar J. Scherer (2000). The preliminary design of a wearable computer for supporting Construction Progress Monitoring, Internationales Kolloquium über die Anwendung der Informatik und der Mathematik in Architektur und Bauwesen, Weimar, Germany, June 2000.

Reinhardt, J., Akinci, B., and Garrett, J.H. Jr. (2002). Using Customized Navigational Models to Deliver more Efficient Interaction with Mobile Computing Devices on Construction Sites, International Symposium on Automation and Robotics in Construction, Washington DC, 2002.

Reinhardt, J. (2003). Navigational Models for Effective and Efficient Interaction with Integrated Product and Process Models on Construction Sites, PhD-Thesis, Carnegie Mellon University, Pittsburgh 2003.

Reinhardt, J., Akinci, B., and Garrett, J.H. Jr. (2004). Navigational models for computer supported project management tasks on construction sites. (submitted and accepted in Journal of Computing in Civil Engineering)

Staub-French, S. and Fischer, M. (2000). Practical and Research Issues in using Industry Foundation Classes for Construction Cost Estimating. Working Paper, Nr. 56, Center for Integrated Facility Engineering, Stanford University.

Wills, G. (1996). Selection: Ways to Say 'This is Interesting', Proc. Information Visualization '96, IEEE, pp. 54-60. 